\title{
AIBR
}

\section{ESTIMADO LECTOR/A:}

Gracias por descargar este artículo. El texto que está a punto de consultar es de acceso libre y gratuito gracias al trabajo y la colaboración desinteresada de un amplio colectivo de profesionales de nuestra disciplina.

Usted puede ayudarnos a incrementar la calidad y a mantener la libre difusión de los contenidos de esta revista a través de su afiliación a la asociación AIBR:

\section{http://www.aibr.org/antropologia/aibr/socios.php}

\section{LA ASOCIACIÓN A AIBR LE PROPORCIONARÁ UNA SERIE DE VENTAJAS Y PRIVILEGIOS, ENTRE OTROS:}

1. Recibir en su domicilio la revista impresa, en Europa y América (tres números anuales).

2. Derecho a voto en las asambleas de socios, así como a presentarse como candidato a la elección de su Junta Directiva.

3. Acceso al boletín de socios (tres números anuales), así como la información económica relativa a cuentas anuales de la asociación.

4. Beneficiarse de las reducciones de precio en congresos, cursos, libros y todos aquellos convenios a los que a nivel corporativo AIBR llegue con otras entidades (incluidos los congresos trianuales de la FAAEE).

5. Promoción gratuita, tanto a través de la revista electrónica como de la revista impresa, de aquellas publicaciones de las que sea autor y que estén registradas con ISBN. La difusión se realiza entre más de 6.900 antropólogos suscritos a la revista.

6. Cuenta de correo electrónico de la forma socio@aibr.org, para consultar a través de webmail o cualquier programa externo.

7. Promoción de los eventos que organice usted o su institución.

8. Opción a formar parte como miembro evaluador del consejo de la revista.

IMPORTE DE LA CUOTA ANUAL: ACTUALMENTE, LA CUOTA ANUAL ES DE 34 EUROS PARA MIEMBROS INDIVIDUALES.

Su validez es de un año a partir del pago de la cuota. Por favor, revise la actualización de cuotas en nuestra web.

MEMBRESÍA INSTITUCIONAL Y DEPARTAMENTAL: Si usted representa a una institución o departamento universitario, compruebe cómo aprovechar al máximo la red de AIBR para su entidad: http://entidades.aibr.org 


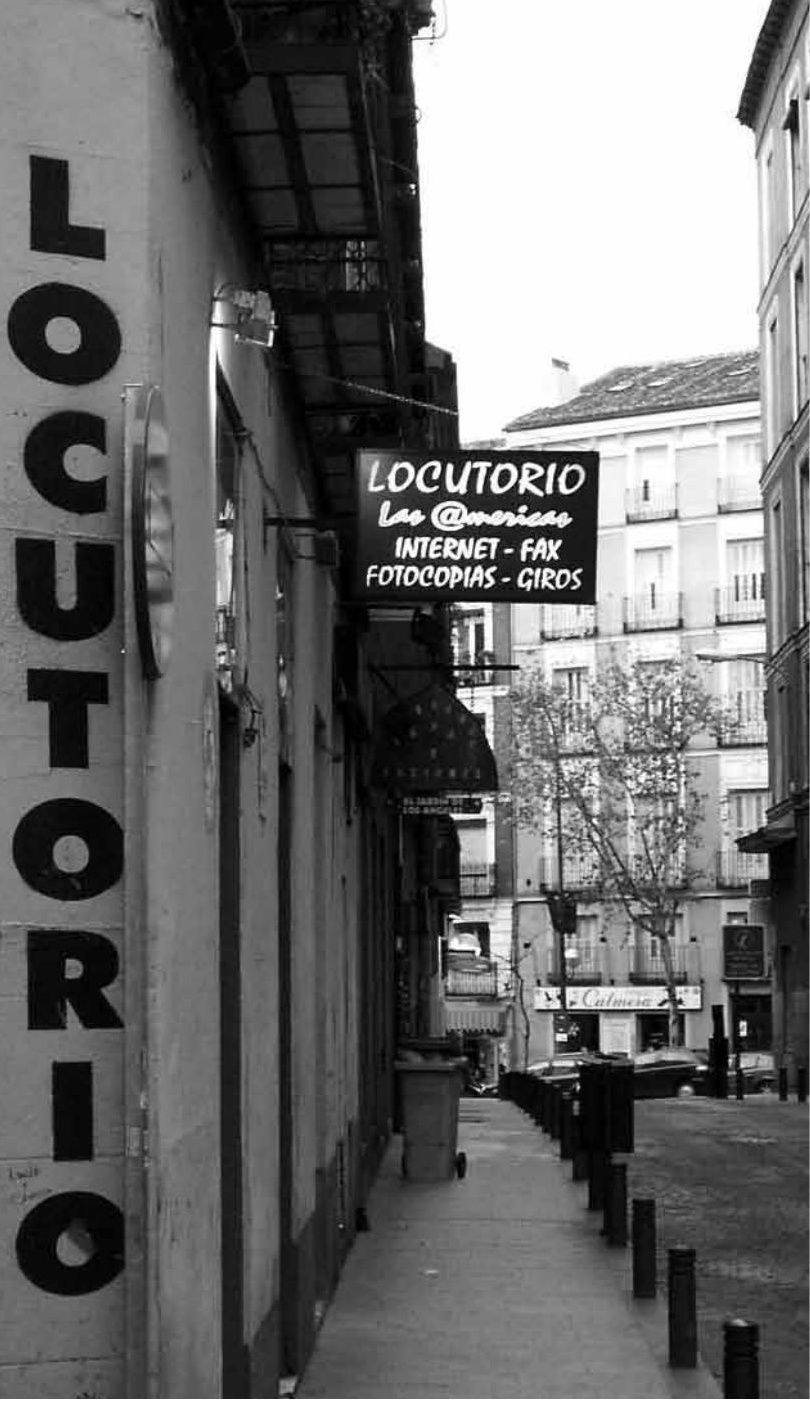

AIBR

Revista de Antropología Iberoamericana

www.aibr.org

VOLUMEN 7

NÚMERO 2

MAYO - AGOSTO 2012

Pp. 197 - 224

Madrid: Antropólogos

Iberoamericanos en Red.

ISSN: 1695-9752

E-ISSN: 1578-9705

\section{COMUNIDADES IMAGINADAS: NACIONALISMO BANAL EN LOS LOCUTORIOS DE BARCELONA.}

JOEL FELIU / UNIVERSITAT AUTÒNOMA DE BARCELONA $M^{\text {a }}$ CARMEN PEÑARANDA-CÓLERA / UNIVERSITAT AUTÒNOMA DE BARCELONA ADRIANA GIL-JUÁREZ / UNIVERSITAT ROVIRA I VIRGILI 


\section{RESUMEN:}

A pesar de la competencia que sufren al multiplicarse las posibilidades de conexión a las redes telefónicas e informáticas, los locutorios de la ciudad de Barcelona y alrededores, no dejan de ser negocios con un relativo éxito. Partiendo de un trabajo de campo colectivo en este artículo articulamos los conceptos de Nacionalismo Banal de Michael Billig y de Comunidad Imaginada de Benedict Anderson, para mostrar que en estos espacios de conexión pueden encontrarse comunidades nacionales imaginadas. La notable vigencia de lo nacional en estos espacios que, paradójicamente, simbolizan como pocos la globalización, no solo no es conflictiva sino que permite la convivencia y facilita la aparición de nuevas comunidades imaginadas.

\section{PALABRAS CLAVE:}

Locutorios; nacionalismo banal; comunidades imaginadas; tecnologías de la información y la comunicación; etnografía; migración.

\section{IMAGINED COMMUNITIES: BANAL NATIONALISM IN BARCELONA “LOCUTORIOS”}

\section{SUMMARY:}

Despite the huge competition that has emerged as a result of the ongoing multiplication of the means to connect to telephone and computer networks, Barcelona call shops, known as "locutorios", are relatively successful small businesses. From a collective ethnographic fieldwork of "locutorios" in Barcelona we articulate Michael Billig's concept of Banal Nationalism and Benedict Anderson's concept of Imagined Community to show that national imagined communities can be found in these public spaces of connection. The remarkable relevance of national identifications in these spaces, which paradoxically symbolize globalization as few do, is not only that it occurs without apparent conflicts but also that it allows coexistence and facilitate the emergence of new imagined communities.

\section{KEY WORDS:}

Call Shops; Banal Nationalism; Imagined Communities; Information and Communication Technologies; Ethnography; Migration. 


\section{Agradecimientos}

Queremos agradecer la colaboración de las siguientes personas en el desarrollo del trabajo de campo: César Jesús Burgos, Gemma Flores, Felipe Corredor, José Gerardo Alvarado, Maria Mena, Albert Farré, Bel Palou, Noel García, Núria Serra y Esther Párraga. También agradecer a los revisores anónimos de la revista su interés y sugerencias.

\section{Introducción}

Hace una quincena de años, Michael Billig introdujo el concepto de $n a-$ cionalismo banal con el fin de describir la forma difusa que toma el nacionalismo en las sociedades contemporáneas, especialmente en los estados-nación consolidados. El nacionalismo banal es un hábito ideológico estrechamente vinculado a la vida cotidiana (Billig, 2006). No se trata de un discurso intermitente, condición de la movilización social para la guerra u otros momentos de crisis, sino de una condición permanente de la vida cotidiana en las naciones establecidas. El nacionalismo banal es un mecanismo omnipresente en estas sociedades, orienta las percepciones y hace aparecer como natural la identificación entre una lengua, una cultura, un territorio y una comunidad política (Billig, 2006). El concepto de nacionalismo banal permite pensar cómo se sostiene la nación en el día a día de entreguerras (o entrecrisis), cuando la exaltación discursiva no está presente pero se mantiene la existencia de la nación mediante prácticas cotidianas aparentemente anecdóticas e insignificantes.

Una década antes, Benedict Anderson había propuesto que la nación debe ser entendida como una comunidad política imaginada, afirmando que "es imaginada porque aun los miembros de la nación más pequeña no conocerán jamás a la mayoría de sus compatriotas, no los verán ni oirán siquiera hablar de ellos, pero en la mente de cada uno vive la imagen de su comunión." (Anderson, 1993: 23). En este artículo, vinculamos ambos conceptos y mostramos cómo estos nos permiten comprender parte del éxito que los locutorios tienen en las ciudades y pueblos receptores de población migrante. Las comunidades, imaginadas en el locutorio mediante prácticas de nacionalismo banal, convierten estos espacios en algo más que locales donde realizar una operación económica, como es la compra-venta de un servicio.

Los locutorios son pequeños negocios a los que la gente acude sobre todo para realizar llamadas o conectarse a Internet; son espacios de conexión donde se concentran, e hibridan, como veremos en este artículo, 
personas, tecnologías, sistemas de comunicación y comunidades nacionales. Si hacemos caso a lo que comentan sus propietarios, los locutorios no son un gran negocio. De hecho, entre sus propietarios y encargados, hay cierto consenso en que uno no se hace rico con un locutorio, e incluso hay quienes afirman que pierden dinero pero que lo mantienen por razones comunitarias, si no románticas. Los locutorios son también una anomalía económica desde el lado del usuario, ya que sus tarifas no siempre son competitivas. Como hemos comprobado, llamar desde un locutorio puede ser más caro que hacerlo desde casa usando una tarjeta prepago de llamadas a larga distancia, como las que se venden en los mismos locutorios y en otros comercios, o incluso acogiéndose a las tarifas especiales que ofrecen algunas compañías telefónicas con la misma finalidad. Por supuesto, no son en absoluto competitivas respecto a la posibilidad de realizar llamadas de voz IP, algo que puede hacerse también desde el mismo locutorio pagando solamente por la conexión a Internet. Entonces, ¿qué tienen los locutorios para que sus usuarios sigan teniéndolos como opción prioritaria para sus comunicaciones?

Una posible respuesta a ambas anomalías económicas yace en el hecho de que en estos espacios, donde gente de diferentes naciones comparte la experiencia migratoria, emergen nuevas redes y comunidades. Aunque en los locutorios se encuentran numerosas muestras de un nacionalismo banal vinculado a las comunidades de origen dominantes en cada uno, éstas no consiguen separar a sus usuarios, sino que son compatibles con el surgimiento de nuevas comunidades imaginarias, supra o extra nacionales. Para mostrarlo, recurrimos al trabajo de campo producido entre 2003 y 2008 en el marco de dos investigaciones ${ }^{1}$ en el transcurso de las cuales visitamos diversos locutorios de Barcelona y sus alrededores. Durante estas visitas realizamos observaciones, tanto participantes como no participantes, y entrevistamos a sus encargados y usuarios. En trabajos previos hemos presentado otros aspectos de los locutorios. Por ejemplo, su imagen pública, vinculada al colectivo migrante, su dimensión económica y a las actividades aparentemente "irregulares" que se daban supuestamente en su interior, fuertemente mediada por los acontecimientos que rodearon al atentado ocurrido en Madrid el 11 de

1. Investigaciones que llevaron por título "Impacto psicosocial y cultural de las innovaciones tecnocientíficas: procesos de cambio y reproducción social vinculados a la implementación y uso de las Tecnologías de la Información y la Comunicación” (SEC2002-03446) e "Impacto psicosocial y cultural de las innovaciones tecnocientíficas: el papel de los espacios públicos de acceso a las TIC en los procesos constitutivos de la identidad social y las redes sociales" (SEJ2006-15655-C02-01/SOCI), subvencionadas ambas por la Dirección General de Investigación (DGI) del Ministerio de Ciencia y Tecnología. 
marzo de 2004 (Garay, Martínez y Peñaranda, 2004); su contribución al mantenimiento y consolidación de vínculos y de relaciones en la distancia con los países de origen y con los otros usuarios con los que se comparte el propio espacio del locutorio (Peñaranda, 2005, 2008); la complejidad y la relevancia de los locutorios en la conformación de la experiencia migratoria (Peñaranda, Vitores, Martínez, Muñoz-Justicia e ÍñiguezRueda, 2011); su carácter vincular y relacional, entendiéndolos como una estación de asociaciones (Íñiguez-Rueda, Martínez, Muñoz-Justicia, Peñaranda-Cólera y Vitores-González, 2012); y finalmente, su contribución a la construcción de las familias transnacionales (Peñaranda, 2005, 2008, 2010; Peñaranda et al., 2011) y de otras redes sociales e identidades (Martínez, Peñaranda-Cólera, Vitores e Íñiguez-Rueda, 2011). Continuando con este proyecto de descripción de los locutorios, nuestro objetivo en el presente artículo es recuperar su carácter y sentido más "nacional" e imaginado, con la finalidad de introducir algunos conceptos teóricos que ayuden a entender el por qué de su éxito.

A pesar de las profecías que auguraban un futuro próximo de homogeneización cultural e identitaria (Amin, 1999; Tomlinson, 1999; Robertson 1992, pero ver también Smith, 1990 para una opinión contraria), poseer una nacionalidad sigue siendo importante para los habitantes del mundo globalizado. Tener nacionalidad no es una opción, no es algo que podamos decidir dejar de tener; aunque podamos optar por cambiar de nacionalidad (cumpliendo ciertos requisitos, nunca sencillos), no podremos optar por no tener nacionalidad. Ser apátrida es siempre un accidente, difícilmente una condición buscada y jamás producto de la voluntad de uno. Por dos razones, una jurídica y la otra simbólica. Jurídicamente, porque existen fuertes restricciones a la posibilidad de vivir fuera de un marco nacional. El pasaporte, o cualquier documento de identidad (aunque sea un acta de nacimiento) es imprescindible para que el estado-nación moderno deje residir en el interior de sus fronteras a los individuos a los que llama ciudadanos en virtud de ese documento, y no los considere, literalmente, un cuerpo extraño. También la posibilidad de participación democrática depende de la documentación que se pueda conseguir. Simbólicamente, porque la identificación moderna entre nación, lengua, cultura, territorio y estado, genera una presión social hacia la defensa de la propia nación como la única manera de salvaguardar idiomas y tradiciones (Feliu, 2004). Se produce, de esta manera, una presión del entorno social para que nos identifiquemos con, y defendamos lo que "es nuestro": "la tierra de nuestros antepasados", "la lengua de nuestros padres"... Esta situación la podemos describir como de naciona- 
lismo globalizado, siendo éste el contexto, jurídico y simbólico, en el que se producen los flujos migratorios actuales.

En este contexto, incluso en las naciones más estabilizadas, sin discusiones identitarias relevantes, la nacionalidad es permanentemente recordada a sus ciudadanos. Michael Billig (2006) muestra cómo un nacionalismo difuso está siempre detrás de los discursos políticos que es posible elaborar en un momento dado, aunque los dirigentes de los estados no pertenezcan a movimientos o partidos explícitamente nacionalistas. Este autor, señala cómo no únicamente se rememora la nación en la política explícita, sino también en los productos culturales, los libros de texto, las calles de los pueblos y ciudades, los balcones de los edificios oficiales, e incluso en la estructura de los periódicos en su clásica separación entre las secciones nacional e internacional (Billig, 2006). A causa de su inocuidad y de su familiaridad, esta rememoración o recreación de la nación pasa desapercibida para la mayoría de los ciudadanos, está integrada en la vida cotidiana. Sin embargo, no es así para los recién llegados, quienes no solamente ven su extranjería recordada constantemente, tanto en las interacciones que deben tener con las instituciones oficiales como en sus relaciones cotidianas en un entorno a menudo hostil, sino que también perciben diariamente esos signos difusos, a partir de una interpelación como "otros", que recrean la existencia misma del país en el que se encuentran. En palabras de Anderson (1993), lo que perciben es el estilo con que es imaginada la nación. En este contexto, los locutorios ofrecen también su propio estilo de (re)crear las naciones, su propio nacionalismo banal. Sus usuarios proceden también de estados-nación, con prácticas nacionalistas banales, y los locutorios son un espacio en los que reencontrarlas. Sin embargo, de la misma manera que son espacios donde se recrean las comunidades nacionales imaginadas, en ellos también se crean nuevas comunidades que giran alrededor del imaginario de la migración.

\section{Los locutorios como espacio tecnológico comunitario}

En los últimos años han surgido en la mayoría de pueblos y ciudades españolas diferentes espacios que ofrecen servicios de acceso a las Tecnologías de la Información y la Comunicación (TIC): bibliotecas, telecentros, locutorios y cibercafés. Estos espacios han venido a cubrir la creciente demanda respecto al acceso y utilización de estas nuevas tecnologías de la información y la comunicación que acompañan al contexto de la globalización (Beck, 1998). Es un contexto insertado en las dinámicas propias 
del predominio de los flujos y la multiplicación de las interconexiones que caracterizan a la sociedad de la información (Castells, 2000). Las bibliotecas y los telecentros, promovidos por la administración pública u organizaciones sin ánimo de lucro, son "espacios físicos que proporcionan acceso público a las TIC para el desarrollo educacional, personal, social y económico" (Gómez, Hunt, y Lamoureux, 1999) y ofrecen estos servicios de acceso tecnológico de manera gratuita. Además del acceso gratuito a las TIC, estos espacios también desempeñan una labor formativa que tiene como objetivo dotar de competencias en el uso de las nuevas tecnologías a aquellas personas que se encuentran en riesgo de "exclusión digital". Es por esto que las bibliotecas y los telecentros tienen un carácter fundamentalmente comunitario, ya que en sus objetivos y sus actividades está muy presente la voluntad de contribuir a paliar los efectos de la brecha digital (Peñaranda et al., 2011). En cambio, los cibercafés y los locutorios son pequeños negocios, por lo que tienen una finalidad fundamentalmente lucrativa. Sin embargo, aunque tengan ánimo de lucro, se trata de espacios que también fomentan la socialización tecnológica y que contribuyen, por lo tanto, al cierre o a la superación de la brecha digital. En la última década, los locutorios se han erigido como los espacios urbanos por excelencia desde los cuales se puede acceder a diversos servicios tecnológicos a precios asequibles.

Hay que notar que, a pesar del gran auge que han tenido este tipo de comercios en el Estado Español, parece que el término (e incluso el propio espacio) locutorio no tiene en sí mismo un carácter global (Martínez et al., 2011). El concepto y el espacio del cibercafé están más extendidos por diferentes países del mundo, como muestran algunos trabajos e investigaciones elaboradas sobre dichos espacios en México (Castro y Zepeda, 2004), Taiwán (Chao et al. 2005), India (Chawla y Behl, 2006), Turquía (Gürol y Sevindik, 2006), Londres (Wakeford, 2003), Reino Unido (Lee, 1999; Liff y Steward, 2003), Botswana (Mauta y Mutula, 2004), Tanzania (Loth, 2001), China (Hong y Huang, 2005), Suecia (Ferlander y Timms, 2006), Escocia y Noruega (Laegran y Stewart, 2003) o Trinidad (Miller, 2004). En cambio, el concepto y el espacio locutorio parece estar más localizado en el Estado Español (Aramburu, 2002; Garay, Martínez y Peñaranda, 2004; Cavalcanti, 2004; Martínez y Peñaranda, 2005; Parella, 2004; Beltrán, Oso y Ribas, 2006; Serra, 2006; Peñaranda, 2005, 2008, 2010) y, con una terminología y uso muy similar, también en otros dos países: en Argentina, aunque casi siempre de la mano del espacio del cibercafé, siendo ambos tipos de espacios objeto de interés para las ciencias sociales que se desarrollan en ese país 
(Schiavo, 2004; Finquelievich y Prince, 2007; Moya y Álvarez, 2007); y en Francia, donde espacios análogos reciben el nombre de téléboutiques o taxiphones (Scopsi, 2002, 2004).

Locutorios y cibercafés comparten muestras de interés por parte del mundo académico. Sin embargo, entre ellos, existen también algunas diferencias que van más allá de su carácter de pequeño negocio tecnológico. Por ejemplo, en España, los cibercafés normalmente sólo ofrecen servicio de acceso a Internet, conjugado con la venta de snacks y refrescos; y, de manera mayoritaria, sus usuarios son jóvenes jugadores de videojuegos o turistas, según la zona de la ciudad donde se encuentren ubicados. Los locutorios, en cambio, son espacios que tienen una mayor oferta de servicios, ya que al servicio de acceso a Internet se le añaden el servicio telefónico, la venta de tarjetas telefónicas de prepago, el servicio de fotocopias y fax, la recarga de móviles, el servicio de envío de dinero y paquetería y, en muchos casos, la venta de comida, snacks y refrescos de importación. Aunque estos servicios no estén dirigidos a una población específica, los locutorios están habitados de forma mayoritaria por población en tránsito y/o población migrante.

Los espacios análogos a los locutorios en París (las téléboutiques o taxiphones, que comparten con ellos la oferta de servicios y la posibilidad de tener un acceso y hacer uso de la tecnología) son caracterizados por Claire Scopsi $(2002,2004)$ como comercios étnicos. Situados en barrios con gran presencia de población migrante, funcionan como espacios de encuentro y de socialización en los que se establecen vínculos transnacionales. A este tipo de comercio a veces se le atribuye cierto papel dinamizador y revitalizador del tejido social de los barrios donde se instalan (Aramburu, 2002). Según Pau Serra (2005: 14) el comercio étnico es "una actividad económica bajo la iniciativa de uno o varios empresarios de origen extranjero y que tiene por objeto la venta de algún producto (bazares, carnicerías...) o servicio (peluquerías, locutorios...) en un local comercial". Otras definiciones atribuyen su carácter étnico al tipo de servicios y productos que ofrecen y a la población destinataria de dichos productos (entendida a priori como un colectivo étnico concreto). Sin embargo, no podemos afirmar, ni mucho menos, que los locutorios sean espacios exclusivamente usados por migrantes, sino que éstos conviven y comparten espacio y servicios con estudiantes, turistas y población autóctona en general ${ }^{2}$. Los locutorios se articulan, por lo tanto, como

2. El término "autóctono" solo debe leerse en este artículo como haciendo referencia a aquellas personas cuya migración ya no forma parte de su historia personal inmediata, sino más bien parte de su historia familiar, más o menos alejada en el tiempo o las generaciones. 
espacios contenedores de personas diversas y de diversas tecnologías, tecnologías que posibilitan la comunicación, el contacto y el intercambio de información. Esta es otra de las grandes características de este tipo de espacios: su carácter de espacio relacional, tanto en el plano presencial como en el plano virtual (Íñiguez et al., 2012; Martínez y Peñaranda, 2005; Gil y Vall-llovera, 2006).

Parella (2004) apunta que, la apertura de este tipo de negocios no tiene que ver con condicionantes de tipo cultural, sino que la ocupación y la auto-ocupación responden a otro tipo de factores de carácter estructural directamente relacionados con las dificultades de la población inmigrante para acceder al mercado laboral de la sociedad receptora. La auto-ocupación, en este sentido, se erige como una forma de supervivencia que está vinculada a los lazos que se tejen dentro de las comunidades. Las redes de dichas comunidades suministran información sobre financiación, contactos y proveedores, facilitan ayuda mutua, pueden contribuir a la formación de futuros empresarios y por supuesto proporcionan fuerza de trabajo barata, tal y como apunta Light et al. (1992, en Parella, 2004). Aunque es discutible que el locutorio sea un comercio étnico, no cabe duda que en estos espacios se materializan redes, no solo en lo que respecta a la búsqueda de trabajo y a la propia posibilidad de ocupación, sino también en otras dimensiones de la vida cotidiana donde se articulan como elemento de apoyo y relación (Íñiguez et al, 2012; Martínez et al., 2011; Peñaranda et al., 2011; Richer y Doré, 2004).

Las informaciones publicitadas en los tablones de anuncios de los locutorios, así como aquellas que circulan en el boca a boca de sus usuarios, son un ejemplo de cómo estos espacios se han convertido en uno de los nodos de la red social de todo migrante. Avisos sobre ofertas y demandas de empleo, alquiler de vivienda y/o habitaciones, ofertas de asesoramiento jurídico y obtención de papeles, publicidad sobre seguros y ocio, etc. son compartidos e intercambiados en el locutorio, haciendo de él un espacio donde ir a buscar información cuando se buscan "pistas" para moverse en la sociedad de acogida y donde conocer estrategias para gestionar la vida cotidiana. El locutorio se articula, por lo tanto, como un punto de paso obligado y, como veremos más adelante, como un lugar de encuentro donde emergen nuevas formas de relación y cohesión social con aquellos que los frecuentan. Esto es algo que también se desprende de las conversaciones escuchadas en los locutorios y de los relatos compartidos de los usuarios: uno va al locutorio a llamar por teléfono o a conec-

En ningún caso debe interpretarse como una posición de más legitimidad respecto a otra en cuanto a su residencia en un país. 
tarse a Internet, sí; pero también para encontrarse con un amigo o vecino, con el que compartir la cotidianeidad, al que pedir consejo frente a un problema o dificultad o simplemente para estar ahí y conversar. Como se puede apreciar en los locutorios, la continuidad de la experiencia migratoria no solo viene determinada por el uso tecnológico, sino también por las dinámicas que se generan, a partir de la apropiación que se hace de estos espacios, en el interior de los mismos (Peñaranda et al., 2011).

Esta caracterización del locutorio como lugar de encuentro, como espacio donde sus usuarios construyen relaciones de amistad y paisanaje, donde se solidifican redes sociales, nos remite a ese carácter relacional en un plano más presencial. Pero no podemos olvidar el plano más virtual, es decir, aquel que, mediado por tecnologías, se establece y mantiene a partir del propio uso tecnológico, y que permite que los usuarios de los locutorios puedan realizar una llamada telefónica, enviar un correo electrónico o una fotografía digital o mantener una conversación por Skype con aquellos que permanecen en los lugares de origen. Estos vínculos y contactos, frecuentes y cotidianos, son los que nos permiten hablar de, por ejemplo, la posibilidad de establecer vínculos transnacionales y, más concretamente, de la posibilidad de hacer familias de larga distancia, o familias transnacionales (Peñaranda, 2005, 2008, 2010; Peñaranda et al., 2011).

En estos espacios, la distancia entre los países se hace patente en cada llamada, en cada envío de dinero o de paquetería. Esta distancia permite que el locutorio emerja como un espacio de articulación simbólica y legal del estado de origen y del estado de destino en el cual se ubica. Al estar físicamente ubicado en un territorio nacional específico, el locutorio se convierte en un espacio donde se proveen servicios de gestoría de documentos oficiales, de transmisión de saberes burocráticos y de descubrimiento de los paralelismos entre los estados nación.

\section{Investigando en los locutorios}

Realizamos el trabajo de campo en distintos locutorios de Barcelona y su área metropolitana entre 2003 y 2008, en el marco de las investigaciones más amplias citadas anteriormente. El objetivo genérico de ambas investigaciones era evaluar las repercusiones psicosociales y culturales que comportan las innovaciones tecnocientíficas asociadas al desarrollo de TIC. De manera más específica, imaginamos que los espacios públicos de acceso a las TIC tenían que tener un papel importante, entendiendo que esta relevancia radicaría no solo en su uso como herramienta de 
comunicación y transmisión de información sino en que éstas estarían participando activamente en la generación de nuevas formas de relación social y de estructuración de redes sociales, generadas virtual y presencialmente, de manera que deberían constituir nuevas identidades personales y colectivas.

La investigación se llevó a cabo mediante el uso de técnicas etnográficas: observación, observación-participante y entrevistas. La opción por una investigación inspirada en la etnografía surgió de la necesidad de trabajar con un tipo de investigación que nos permitiera completar las formas habituales de trabajo mediante entrevistas, típicas de la psicología social de corte cualitativo de los últimos años, con la descripción de los espacios investigados y sus usos. Esta modalidad de trabajo nos ofrecía al mismo tiempo la posibilidad de interpretar las situaciones que en estos se generan y, por lo tanto, una oportunidad para comprenderlas. La etnografía supone, más que un conjunto de técnicas que nos permiten recoger datos, un modo de encarar el mundo (Taylor y Bogdan, 1984): a) por la atención que se le presta a los significados y percepciones de las personas objeto de estudio en contextos sociales específicos; b) porque se llevan a cabo tareas de tipo observacional, descriptivo, interpretativo y comprensivo que nos permiten dar cuenta de los sentidos de las prácticas de los administradores y usuarios del locutorio y, c) porque tiene un carácter inductivo que se centra en un contexto concreto y reconoce el potencial de la investigación hecha en localizaciones específicas. Estos aspectos nos resultan especialmente relevantes para indagar sobre los sentidos nacionales y/o imaginados que las personas dan a los locutorios, los objetos que contienen y las comunidades que los habitan.

La búsqueda de información sobre la presencia de locutorios en Barcelona y Cataluña conllevó un periplo por diferentes bases de datos y organismos, así como por los diferentes servicios de las administraciones locales y autonómicas (la Cámara de Comercio, las Sedes de los distritos de la ciudad, el Servicio de Urbanismo del Ayuntamiento, el Instituto de Estadística de Barcelona y el Instituto de Estadística de Cataluña), los cuales en ningún caso nos proporcionaron un número ni exacto ni aproximado de los locutorios existentes en Barcelona y su área metropolitana. A falta de registros específicos, intentamos acceder de otra manera a dicha información a través del Impuesto de Actividades Económicas (IAE) y su registro de petición y concesión de licencias de apertura. La información proporcionada en dicho registro presentó, a su vez, muchas limitaciones ya que, al no haber un código específico para la actividad de los locutorios, éstos podían incluirse en varias categorías de licencia 
de apertura (como por ejemplo, el código 761: Servicios telefónicos o el código 769: Otros servicios de telecomunicación. También nos dimos cuenta que algunos locutorios se registraban con frecuencia bajo el código 999: Otros servicios NCAA -no comprendidos en otras categorías-). El que los locutorios pudieran estar registrados en varias categorías, así como la falta de garantía respecto a la actualización de la información, hizo patente la imposibilidad de conocer a ciencia cierta la cantidad de locutorios que existían en Barcelona en esos momentos. Incluso, cuando el servicio de urbanismo del Ayuntamiento de Barcelona nos remitió un documento donde nos detallaba el número de cibercafés y locutorios existentes por cada distrito de la ciudad de Barcelona, información basada en la tramitación de licencias de actividad, encontramos muy poca correspondencia entre la cantidad de locutorios contabilizados en ese documento y los que habíamos observado en nuestro caminar por los barrios durante la fase de trabajo de campo. A esta dificultad de acceso a la información sobre los locutorios, también hace referencia Serra (2005) en su trabajo sobre el comercio étnico de Ciutat Vella. Serra resuelve este problema realizando un "recuento a mano" de los diferentes comercios étnicos. A título de ejemplo, este investigador afirma que en 2004 había 31 locutorios en el distrito de Ciutat Vella de Barcelona. En nuestro caso, y al abarcar nuestra investigación un territorio considerablemente mayor, decidimos proseguir sin ese dato y finalmente, el trabajo de campo tuvo lugar en 16 locutorios de diferentes barrios de Barcelona (Ciutat Vella, Sant Antoni, Fort Pienc, Sants, Poble Sec, Eixample y Gràcia), así como en varios municipios del área Metropolitana de Barcelona (específicamente, en Cerdanyola del Vallès, Sabadell y Castellar del Vallès), seleccionados por cada investigador en función de criterios de familiaridad, accesibilidad y/o heterogeneidad de la muestra. A partir de los contactos realizados en ellos se realizaron 27 entrevistas abiertas.

En el trabajo de campo, a parte del personal investigador del departamento de Psicología Social de la Universitat Autònoma de Barcelona adscrito a los grupos GESCIT $^{3}$ y JovenTIC ${ }^{4}$, también participaron estudiantes del último año de la licenciatura de Psicología, estudiantes del Máster Oficial de Investigación en Psicología Social y estudiantes de los Estudios de Doctorado en Psicología Social. Todos ellos fueron formados previamente en técnicas cualitativas de recogida e interpretación de datos,

3. Grupo de Estudios Sociales de la Ciencia y la Tecnología (http://psicologiasocial.uab. cat/gescit).

4. Joves i Tecnologies de la Informació i la Comunicació (http://psicologiasocial.uab.cat/ joventic). 
tanto en asignaturas de sus estudios como en seminarios ad-hoc. Durante el período de trabajo de campo se realizaron seminarios de investigación semanales en los que se comentaban las incidencias del día a día en los locutorios, tanto en cuestiones de interacción en el campo como, por ejemplo, respecto a las estrategias de inserción y de contacto con los usuarios y encargados, con la toma de notas, el redactado de los diarios de campo o las transcripciones de entrevistas. Debido a la diversidad de perfiles vitales y características personales de los investigadores que realizamos el trabajo de campo, las formas de estar presentes en el locutorio fueron diversas. En algunos casos, se trató de investigadores latinoamericanos que investigaron en locutorios de los que ya eran clientes previamente y que simplemente explicitaron su nueva condición al empezar el trabajo de campo. Cuando no eran clientes del locutorio con anterioridad, su integración en el locutorio se produjo de forma relativamente fluida ya que podían identificarse como investigadores pero también como usuarios simultáneamente, así como explicitar una situación vital parecida a la de otros clientes o incluso a la del personal que regentaba el locutorio. En otros casos, cuando se trató de investigadores que no requerían el uso de los servicios del locutorio de forma habitual ni compartían una experiencia migratoria personal, la negociación del papel a desarrollar en el locutorio fue más dificultosa, dado que el investigador sentía que su presencia continuada ahí no era esperada. Al final, en casi todos los casos la situación se resolvió sin graves problemas y la presencia de los investigadores fue bien aceptada, en tanto que los usuarios y encargados de los locales mostraron en general una buena disposición hacia la investigación planteada, a la que parecieron recibir como una oportunidad de compartir su experiencia migratoria. Las entrevistas muestran el especial interés de los migrantes en explicar sus vivencias, tanto aquello que tiene que ver con su vivencia de la distancia y de la separación de sus seres queridos, como las dificultades con la burocracia, el trato y recibimiento de la población local o las complicaciones económicas del día a día.

\section{La comunidad nacional imaginada en el locutorio}

La identidad nacional no puede ser pensada circunscrita únicamente a un territorio geográfico concreto. Los procesos migratorios y diaspóricos han generado que haya naciones que tienen una gran cantidad de ciudadanos en el extranjero, dispersos en diferentes latitudes geográficas. En nuestro trabajo de campo vemos, por ejemplo, como el locutorio puede ser una extensión del territorio nacional en la que participar de la política 
del país. Los usuarios de un locutorio regentado por una pareja mixta dominicana-catalán, hablan de política y de las elecciones por venir en su país, y lo hacen con sumo detalle, por ejemplo discutiendo sobre las atribuciones de la Junta Electoral Central. Los dominicanos en el exterior pueden votar en las presidenciales, y hay oficinas para inscribirse habilitadas en EEUU, Puerto Rico, España (Madrid y Barcelona), Venezuela y Canadá. En el locutorio se comenta si el presidente actual es bueno o no, y se percibe cierta movilización y ánimo de participar. La encargada y los clientes hablan de política muy abiertamente y en voz alta, discuten durante largos minutos sobre corrupción, justicia y buen gobierno.

Hablar de nación no es hablar, por lo tanto, de un espacio geográfico concreto, sino de una nacionalidad extendida y, en esta extensión, se producen reconocimientos y exaltaciones, pero también negociaciones, sincretismos e integraciones a través de la participación de la diáspora. Anderson (1993) caracteriza esta situación como de nacionalismo a larga distancia, hasta el punto que la vinculación nacional de un locutorio puede ser literal, como en el caso del locutorio de la calle Floridablanca de Barcelona que fungió de extensión física del consulado boliviano, en un momento en que éste se vio desbordado por la cantidad de gestiones que tuvo que realizar por el aumento de la llegada de bolivianos a la región. O puede ser figurada, como en el locutorio del barrio de Sants donde se promueven conciertos de música colombiana, al tiempo que se participa directamente en la organización del día de la independencia de Colombia, así como también se publicitan negocios tan variados como una clínica dental, una sala de fiestas, incluso cursos de baile o de cocina colombiana, etc. De un modo parecido, de entre la diversidad de servicios que se ofrecen en el primer locutorio citado, se encuentra el apoyo a personas dominicanas (como la propietaria) en la búsqueda de empleo o en el asesoramiento jurídico-legal.

Todo ello son muestras de nacionalismo banal que reproducen y sostienen, literal y figuradamente, la existencia de algo como Colombia o la República Dominicana. Evidentemente, la política y la burocracia que producen los gobiernos acreditan de su existencia, pero las actividades mencionadas muestran también la conformación de una comunidad imaginada alrededor de la idea de un estado-nación de origen.

Billig (1998) argumenta que el nacionalismo banal reproduce cotidianamente los esquemas mentales del nacionalismo, ya sea en rituales colectivos como el deporte, o en detalles nimios como la utilización de banderas para identificar las lenguas en las que se escriben los ingredientes de una caja de cereales. La existencia de naciones es un dado por 
hecho compartido entre quién regenta el locutorio, sus usuarios y los propios investigadores, que lo inscribimos en nuestros diarios de campo:

Locutorio Latino es el nombre de este lugar (...). En la decoración del locutorio se pueden observar banderas de países latinoamericanos y otros objetos característicos de diferentes regiones del mundo, por ejemplo: platos con imágenes de Venecia, transportes de Venezuela, muñecos con vestimenta de Cuba, bailarinas de Brasil, un mapa de Colombia..." (Diario de campo de investigador 3).

La aparición de Venecia en este extracto nos recuerda que la nación no es un hecho natural, sino una construcción social relativamente arbitraria. Otros referentes son posibles pero más improbables: por ejemplo, aunque los transportes, muñecos o bailarinas sean probablemente de una región concreta de su país, son usadas como metonimia visual del país entero. A través del concepto de nacionalismo banal, Billig llama la atención sobre las formas del nacionalismo que podemos encontrar más próximas y que nos resultan tan familiares que las solemos dar por hecho. Por ejemplo, muchos locutorios tienen relojes con horas de países diferentes. En uno de ellos, tres relojes de pared están marcados cada uno con los nombres de algunos países (es de suponer que los relevantes para la clientela): el primero, Perú, Colombia y Ecuador; el segundo, Senegal y Marruecos; el tercero, Bolivia y Paraguay. En los locutorios, la imagen de los relojes con las horas de diferentes ciudades, típica de una bolsa de valores, un hotel de lujo o de las oficinas de una empresa multinacional, ha sido reemplazada por horas nacionales; en éstas una nación equivale a una franja horaria, sumándose a la aspiración real de los estados extensos que han elaborado políticas explícitas de unificación horaria dentro de su territorio, como es el caso relativamente reciente de Venezuela.

Para Billig (2006), la mayor parte del nacionalismo que nos envuelve no es un nacionalismo agresivo sino tan banal como el aire que respiramos y, por lo tanto, tan difícil de ver como éste. En un momento dado, uno de nosotros, al salir de la cabina y pagar por el tiempo de uso, se dio cuenta de que en el mostrador tenían pulseras a la venta, pulseras tejidas con hilos de colores y con diferentes nombres de personas, países y signos. Después de averiguar el costo e interesarse por si las traían de algún lugar, al averiguar que la hacía el encargado, pidió una pulsera con los colores de la bandera de México y con el nombre de uno de sus compañeros de piso, originario de ese país. Billig argumenta que cualquier defensa, demostración o incluso simple muestra de la existencia 
de una nación, puede ser considerada una muestra de nacionalismo, al reiterar repetidamente en los gestos cotidianos más anodinos, un proceso de materialización de una comunidad que ante todo es imaginada. Los locutorios están impregnados de imaginarios nacionales: banderas, fotografías de lugares emblemáticos, pósters, productos alimenticios, etc. son algunos de los elementos que están presentes de forma reiterada y que nos informan generalmente del origen del propietario y/o encargado del locutorio, actuando como referentes o representantes metonímicos del país de origen. Esta apropiación simbólica de la nación incluye en ocasiones los fondos de escritorio y los salvapantallas de los ordenadores, donde también se pueden encontrar fotografías y/o nombres característicos del territorio original. En algunos locutorios también podemos encontrar pequeños colmados donde se venden productos y alimentos del lugar de origen. La música ambiente suele ser también originaria del país del encargado del locutorio, aunque pocas veces se trata de música tradicional, sino más bien de composiciones modernas que nos remiten al carácter presente de la nación (por ejemplo, en uno de los locutorios cuyo encargado era paquistaní, siempre tenían puesta música moderna producida en el subcontinente indio). Estos objetos (las fotografías, los pósters, las banderas, la comida, la música, etc.) actúan como marcas identitarias que hacen referencia al país de origen, y actualizan y rememoran la nación, no solamente en el espacio físico del locutorio sino también en las emociones de sus usuarios y usuarias:

y la música... es como un domingo... tu ven... porque en el domingo hacen comida aquí de mi país... aquí de Perú... yo vengo un domingo_ponen música de mi país...y me transporto...como si estuviera un domingo en casa... o ahí en un sitio... en un restaurant... de mi país....y me relajo y me lo paso... escucho música... y de ahí que me apetezca tomarme una clarita...y comes algo de tu país... eso que la gente que se queda ahí... pues mira... sigues sufriendo (investigador 4, entrevista 2).

A través de la rememoración/conmemoración y de los referentes de pertenencia a un lugar o a una comunidad, el locutorio se convierte en un espacio de (re)construcción de la nación (Garay, Martínez y Peñaranda, 2004). Dicho de otro modo, la nostalgia que se muestra en el relato de esta mujer a través de la imagen del sufrimiento y del transporte provocado por la comida y la música, aviva el sentimiento de la existencia de algo como "tu país". 
Dicho esto, no podemos olvidar que los locutorios son nacionales en dos sentidos: en primer lugar, como acabamos de decir, por ser espacios de recreación de la nación de origen de quien lo atiende o de sus usuarios mayoritarios; y, en segundo lugar, por estar físicamente ubicados en el territorio atribuido a una nación, llamada eufemísticamente de acogida. Esas dos (o más, en ocasiones) nacionalidades del locutorio se articulan entre sí en ese espacio.

Entonces aquí [en el locutorio] es un punto donde consigues muchísimas cosas y particularmente, también lo que digo mucho, es que se consigue trabajo. Por ejemplo, una persona que ya trabaje en una casa de familia, en el caso de las mujeres, cuando ya conocen a esa chica ecuatoriana, peruana, colombiana... bueno, venezolana, como sea, a través de ella, le recomiendan conseguir alguna persona para algún familiar de un patrón español o, de aquí, una familia catalana, entonces, “iMire! Consígueme una chica como tu” y esta trae a otra y a otra... y así se consigue empleo. A una chica que ya la conozcan por referencia... ella ayuda a conseguir otro empleo recomendando a otros emigrantes (investigador 5 , entrevista 1 ).

Destaca de este extracto que, las personas que buscan trabajo, así como las que buscan trabajadoras, son caracterizadas, en primer lugar y casi como único atributo, por su nacionalidad. Pero en él también se muestra el locutorio como un espacio donde se ponen en contacto simbólicamente ambos grupos de nacionalidades (de aquí o de allá). Incluso puede notarse la elaboración que supone un reconocimiento nacional complejo al matizar que puede buscarse alguien para "algún familiar de un patrón español o, de aquí, una familia catalana”.

Paradójicamente, si se quiere, y siguiendo con el análisis del mismo extracto, esta identidad nacional es con frecuencia una identidad inmigrada que actúa como aglutinadora de personas que comparten una experiencia migratoria. De esta forma, el locutorio se articula como espacio nacional y transnacional, donde la identidad nacional, compartida o no, se convierte en una excusa para compartir la experiencia migratoria. Esta experiencia facilita no sólo la vivencia, sino también la convivencia, a partir de los objetos mencionados se da un espacio de construcción de imaginarios nacionales compartidos, aunque no siempre comunes. A los imaginarios nacionales propios de las comunidades del locutorio se le agregan los imaginarios que circulan sobre la nación de llegada, compartidos con el resto de usuarios, sean nacionales del país que sean: 
De papeles también...cuando yo hice lo de la renovación de mis papeles... les... de... yo le pregunté a Marlene... y alguien... yo que sé. Mario o cualquier persona... de los que estaban ahí “ah sí porque se necesita esto, se necesita lo otro... pero tienes que ir aquí” o “oye... mejor vete acá al SCAI y pregunta bien”... el común que tenemos entre nosotros, pues no sé... creo que nos ayudamos...un poquito... (investigador 4, entrevista 1).

Los subrayados del extracto anterior destacan la emergencia de una comunidad "el común que tenemos entre nosotros" y que dentro de esta comunidad las personas son intercambiables entre ellas, pues cada una representa la comunidad: "Mario o cualquier persona de los que estaban ahí”. Se comparten las estrategias que las personas siguen para gestionar su presencia en la sociedad de destino y, al tiempo, se nos muestra cómo manejan su reconocimiento mutuo (Garay, Martínez y Peñaranda, 2004). El locutorio es un espacio donde se habla de la gestión de aspectos administrativos y/o burocráticos, de manera que, como nos explican, uno puede enterarse de la documentación que necesita (fotocopias, pasaporte, documentos, etc.) para realizar diferentes trámites, como por ejemplo el proceso de reagrupación familiar que padres y madres inician para traer a sus hijos e hijas consigo, que se menciona a menudo.

Destaca que el reconocimiento mutuo pasa por reconocer al otro por su nación, al referir anécdotas de gente que ha encontrado trabajo o ha conocido gente en el locutorio, el descriptivo más habitual es la nacionalidad, "un chico de Perú", "un señor de Argentina”, etc. Así pues, de manera banal cada persona es vista y presentada en primer lugar por su origen nacional. Es precisamente el carácter no conflictivo de esta identificación lo que muestra y garantiza a la vez su potencial naturalizador: cada uno de nosotros pertenece a un país y no pasa nada. Si no es como deben ser, al menos así es como son las cosas.

En un contexto de nacionalismo banal la idea de una comunidad imaginada no es baladí, al contrario, el imaginario compartido del hecho de provenir del mismo estado es poderoso y reconfortante, en palabras de los usuarios. Como se menciona en una entrevista, la idea de encontrarse con alguien de Sudamérica es agradable, aunque "si es peruana, mejor". Y cuenta la entrevistada que reconforta porque suple la familia que no está. El discurso nacionalista que presenta la patria como una gran familia se incorpora especialmente en los afectos de quién migra, y de quién, por lo tanto, puede sustituir más fácilmente una metáfora familiar basa$\mathrm{d} a$ en el parentesco por otra basada en el estado-nación de procedencia. 
Esa comunidad puede realizarse a través de la percepción de un lenguaje compartido ("éramos más afines a hablar, sabíamos, no sé, una jerga que digas... pues te la entienden..."), o bien de unos sentimientos comunes ("el mismo sentimiento", "me entenderían exactamente como me siento... como es la misma posición”), que provocan la aparición de un otro igual, opuesto, en el siguiente extracto, al intimo diferente:

es que como mi novio solo habla con...mi novio tiene su mundo... y es muy lejos de los locutorios y de gente suramericana... emm...para con gente muy catalana... y ellos tienen su mundo (investigador 4 , entrevista 1).

En realidad, lenguaje y sentimientos son las dos caras de una misma moneda. En ambos se expresa la idea del confort social que supone reconocer en el otro la igualdad con uno, una igualdad imaginaria pero con efectos muy concretos, como la posibilidad que tu interlocutor te entienda sin excesivos rodeos o explicaciones. Esta comprensión ocurre por la similitud de la experiencias vividas ("hemos vivido lo mismo... o vivimos lo mismo..."), que provocan afectos similares ("los sentimientos son muy parecidos...los sentimientos son muy, ,muy, muy parecidos... ), que contrastan con los que puede tener la gente autóctona ("en cambio aquí no te entenderían...o...o...te entienden...pero...no es...no lo sienten...no lo sienten...", “como no lo han vivido...no lo sienten...creo que es eso...”). Este contraste es fundamental para comprender cómo el desconocido cousuario, el otro igual, del locutorio se convierte en virtud de una comunidad imaginada compartida en alguien que "ha vivido lo que tú “ y que te permite retornar a tu yo "original", compensando la nostalgia sentida "aunque sea cinco minutos" por volver a "hablar como hablabas allá".

\section{Nuevas comunidades imaginadas en los locutorios}

Lo expuesto hasta ahora no trata de mostrar el funcionamiento de la categorización nacional, o étnica, en los locutorios sino una muestra de cómo se construye en estos espacios una diversidad de comunidades a través del uso del nacionalismo banal, lo que no quiere decir que los locutorios sean un espacio nacionalista. De hecho, cualquier adscripción étnica o nacional de éstos presenta numerosas limitaciones que imposibilitan que se configuren como un espacio discursivo nacionalista. Estas limitaciones surgen al menos por tres razones: la nacionalidad de su titular, la heterogeneidad de su clientela y el surgimiento de nuevas comunidades 
en estos espacios. En primer lugar, el origen geográfico de su propietario no es una evidencia. Aunque existe la creencia compartida de que los propietarios de los locutorios son extranjeros y/o inmigrantes, existen locutorios cuya propiedad es catalana y/o española, tal y como nos dijeron las personas que los regentaban. Como indica Serra (2005), el hecho de que la titularidad de los locutorios (y por ende, de los comercios étnicos) sea extranjera no se puede generalizar.

En segundo lugar, aunque es cierto que algunos comercios parece a primera vista que van dirigidos exclusivamente a una clientela mayoritariamente inmigrante (incluso podríamos decir que a un colectivo étnico concreto), como podría ocurrir con las carnicerías halal, con ciertas peluquerías o incluso con los propios locutorios, estos espacios son frecuentados por una clientela diversa. Como apunta Aramburu (2002), algunas carnicerías halal o ciertas peluquerías árabes o caribeñas son frecuentadas por autóctonos ya sea por cuestiones de vecindad, por aprecio de sus productos o servicios o por sus precios económicos. Lo mismo ocurre con los locutorios ya que, aunque hayamos podido categorizarlos como "el locutorio dominicano", "el locutorio paquistaní, etc. en nuestros diarios de campo (ya sea porque el regente del locutorio tenga esa nacionalidad o porque los elementos que decoran el espacio connoten esta nacionalidad), los servicios y productos que ofrecen no se dirigen a una población específica. Por ejemplo, un locutorio regentado por dominicanos no limitará la oferta de sus llamadas a la República Dominicana, sino que englobará en sus ofertas a otros países y/o áreas geográficas. Los locutorios, por el tipo de servicios y productos que dispensan, no están destinados a priori a una comunidad étnica o a una nacionalidad específica, sino que se dirigen más bien "a una clientela transversal en lo nacional y lo religioso" (Aramburu (2002: n/p). Esto es, se orientan más bien hacia la satisfacción de las nuevas demandas del conjunto de la población, inmigrante o no. En esta heterogeneidad, que hemos podido comprobar en nuestro trabajo de campo, los autóctonos son también clientes que se acercan a este tipo de espacios para, al igual que la población migrante y/o en tránsito, hacer uso de las tecnologías de la información y la comunicación (estudiantes que navegan, chicos que juegan, chicas que chatean, turistas que usan el correo electrónico, amas de casa que recargan móviles, etc.).

En tercer lugar, las redes sociales que se generan a partir de la conexión que facilitan las TIC, no solo generan la recreación de una comunidad imaginada, sino que en ellas se producen una multiplicidad de comunidades imaginadas. En los locutorios no sólo se recrea la comunidad 
imaginada específica de un lugar de origen concreto, sino también otras "comunidades imaginadas" como pueden ser las que remiten a la región: Asia, África, el Caribe, América Latina o Europa. Un ejemplo de cómo se vincula la experiencia de ser migrante con la creación de comunidades imaginarias de más amplio alcance que las nacionales, lo encontramos en los locutorios orientados a clientes indios o paquistaníes. A pesar de los enfrentamientos en origen entre paquistaníes e indios, en estos locutorios existe una buena relación entre unos y otros. Esta relación no conflictiva, se debe sin duda a que, en Barcelona, ambos colectivos comparten el hecho de ser migrantes, se encuentran con dificultades similares, ligadas al rechazo o la estigmatización, y por lo tanto pueden encontrar también esta comunidad de sentimientos a la que aludíamos anteriormente. La comunidad imaginada nacional queda difuminada en pos de una comunidad imaginada regional (ese compartir dificultades, rechazo por parte de la población autóctona, etc.) prevaleciendo, en la interacción en el locutorio (y es posible que en otros contextos de interacción) sobre la otra, posibilitando el contacto, la relación e incluso la amistad.

Por estas razones, el adjetivo étnico no caracteriza de forma suficiente estos espacios. Tampoco el adjetivo nacional, aunque es cierto que ambos se corresponden claramente con al menos parte importante de las prácticas que tienen lugar en los locutorios. Este carácter étnico/nacional hace más bien referencia a la simbología (fotografías, banderas, música, pósters, salvapantallas, etc.) que hemos encontrado en los locutorios; simbología que ejerce de elemento aglutinador para ciertos colectivos, conformando la comunidad imaginada. Muchos de los trabajos elaborados en torno al concepto de comercio étnico no pretenden indagar sobre las implicaciones ideológicas y sociopolíticas del término étnico como categoría social, y tampoco es el objetivo de este trabajo hacer lo mismo con el término nacional. A pesar de que son problemáticas, estas conceptualizaciones nos permiten entender mejor lo que acontece en los locutorios. Pero hay que tener cuidado con las implicaciones sociopolíticas del uso de los adjetivos étnico y nacional, ya que pueden contribuir a la reificación de ciertas representaciones recurrentes sobre los inmigrantes y sus actividades (Aramburu, 2002). De hecho, las comunidades imaginadas no sólo se conforman con el significado que se trae de la comunidad de origen, sino también con las interacciones y sincretismos que se producen en los lugares de destino, donde no sólo coinciden con personas de su mismo origen nacional (pudiendo compartir ciertas afinidades, similitudes, etc.) sino que también coinciden con otros migrantes (de otros destinos nacionales) y con autóctonos, con los que tienen que 
negociar también múltiples significados, entre ellos los relacionados con la nacionalidad, y especialmente con los estereotipos y prejuicios, cuya negociación implica un juego constante de confirmación y/o negación de lo que uno "es". Por ejemplo, algunos argentinos que no son de Buenos Aires se encargan de dejar muy clara cuál es su procedencia (“...pero yo soy de Córdoba“) para distanciarse del argentino porteño y de los estereotipos que, ellos mismos, entre otros, suelen asociar con los argentinos que proceden de la capital.

\section{Conclusiones}

Ciertamente, el nacionalismo banal local no es tan banal para los inmigrantes. Por ello, el nacionalismo del locutorio se podría entender también como un nacionalismo de oposición. Como comenta Billig (2006), en un mundo internacional, es importante que las naciones no se confundan unas con otras ni se solapen, sino que se mantengan lo suficientemente alejadas simbólicamente unas de otras para preservar su diferencia. Este discurso nacional puede constatarse en algunas prácticas cuidadosas de preservación de las costumbres, ritos, acentos, etc. que pueden leerse en términos de oposición, de diferenciación respecto al autóctono, de reconocimiento de su propia identidad y de aquellas características que les permiten diferenciarse y ser identificados como tales (cosa que le permite a uno sentirse localizado, perteneciente a un lugar concreto). Estas marcas de identidad son a veces interpretadas como problemas de integración en la sociedad de acogida o como una especie de repliegue identitario que dificulta, a su vez, esta integración, pero De la Haba y Santamaría apuntan que

En entornos urbanos, la constitución de (auto) dominios específicos (incluso marcados étnicamente) es un modo inmediato de acomodación del que disponen los grupos en situación de cohabitación pluricultural. En estos contextos, la afirmación identitaria sobre el espacio público, sobre sus objetos y referentes físicos, no debe ser interpretada de forma unívoca como una negación de la coexistencia, sino más bien como un reflejo de las distancias sociales o culturales, y sobre todo, como un modo - variable, inacabado y sujeto a permanentes reformulaciones o compromisos - de gestión de los alejamientos y reconocimientos socioculturales. (2004: 129)

En este sentido, las marcas étnicas o identitarias que caracterizan a los 
locutorios, lejos de representar una falta de integración o de resistencia a los valores de la comunidad de acogida, nos muestran cuales son las estrategias que siguen las personas migrantes al intentar participar de un modo de vida que suponga un reconocimiento identitario, algo que según Taylor (1992) es una necesidad para la constitución del self moderno que se encuentra inserto en un mundo multicultural, pero también algo que muestra de facto que el sujeto está ya integrado pues intenta vivir su pertenencia nacional en un entorno nacional extraño, apropiándose entonces el nuevo espacio (Delgado, 1998).

El desarraigo que pueda sentir un migrante al abandonar su país y la melancolía resultado de la vivencia de la distancia, así como el extrañamiento por las experiencias en una sociedad nueva, pueden relativizarse "por la experiencia de continuidad en la identidad que proporciona el estar inmerso en una red, una red donde te encuentras con iguales con los que compartir la distancia, la movilidad y la vivencia del migrante” (Garay, Martínez y Peñaranda, 2004: 6). Ambos aspectos (la identidad nacional compartida y el acceso a informaciones vitales para el inmigrante) contribuyen de forma importante al éxito de los locutorios, a que éstos se articulen no solo como espacios contenedores de tecnologías, sino también como espacios donde los migrantes se encuentran y comparten sus experiencias y preocupaciones. Los locutorios no solo están generando relaciones con los lugares de origen mediadas por las tecnologías, sino que estos espacios se convierten en un lugar de encuentro para la población migrante, donde las relaciones que se generan están atravesadas por la proximidad física y por el hecho de compartir un espacio y no solo por artefactos tecnológicos. La continuidad, la variación o la fluctuación en la identidad no es, por tanto, fruto únicamente del uso tecnológico, sino que también tiene que ver con las dinámicas que se generan en el interior de espacios como los locutorios y de las apropiaciones que, de estos espacios, elaboran sus usuarios transformándolo en un lugar de encuentro y reunión (Peñaranda, 2005). Aparentemente, parte del éxito de los locutorios proviene de la creación de un espacio donde todos comparten algo: la experiencia migratoria junto con la experiencia de pertenecer a la misma comunidad imaginada primero nacional y luego regional.

La globalización no parece producir, de momento, un efecto homogeneizador en las poblaciones, más bien parece lo contrario: el hecho de que la globalización ocurra en un contexto de nacionalismo (banal) globalizado multiplica los espacios y los territorios en los cuales se performa la nacionalidad. Cada performación de una nacionalidad renueva su vigor. Sin embargo, al mismo tiempo, estas identificaciones nacionales 
pierden fuerza en el sentido nacionalista tradicional de una identificación fuerte, opuesta a otras naciones, conflictiva, vituperante y chovinista. En el locutorio, las muestras de identidad nacional se viven sin conflicto aparente. Ello no conlleva que esta identificación sea "débil", al contrario, en el locutorio, al igual que en gran parte de la vida cotidiana de una persona migrante, el primer rasgo identificativo que se usa es la nacionalidad. Esa es la que te otorga "ser", lo primero que un otro reconoce de ti.

Las técnicas etnográficas nos han permitido indagar en los sentidos nacionales imaginados de objetos, personas y comunidades y al mismo tiempo evitar la reificación de dichos sentidos. Ofrecen un retrato dinámico de lo nacional imaginado, especialmente en su interacción con lo "local" y el "otro migrante", que nos deja ver el proceso por el que pueden aparecer nuevas comunidades o redefinirse las viejas. La ciudadanía de un país se performa en el locutorio en el momento en que se participa de redes y se expresan emociones (Belli y Gil, 2009). En este artículo hemos articulado los conceptos de comunidad imaginada y de nacionalismo banal para ofrecer una descripción de un espacio urbano contemporáneo. Ambos son conceptos complementarios, que no describen lo mismo pero que se necesitan para poder ofrecer una visión compleja de las relaciones que se viven en el locutorio: un espacio de articulación de emociones, experiencias e identidades. El nacionalismo banal permite la comunidad imaginada: a partir de la percepción de un elemento nacional banal, como una postal con una pequeña bandera en un lateral, emerge la comunidad imaginada. Toda muestra de recreación de una nación (por ejemplo, mediante objetos de uso común) refleja las representaciones más generalistas de la nación, que no la representa toda sino a aquello más vulgarizado, más conocido, mediado, a menudo, por el mercado turístico. Aun así, los objetos del nacionalismo banal pueden servir de excusa para compartir la experiencia migratoria y para facilitar la convivencia al crear un escenario de construcción de nuevas comunidades supraordenadas. En definitiva para generar una identidad compartida en términos de comunidad imaginada (inter)nacional. 


\section{Referencias Bibliográficas}

Amin, S. (1999) [1997]. El capitalismo en la era de la globalización. Barcelona: Paidós. Anderson, B. (1993) [1991]. Comunidades imaginadas: Reflexiones sobre el origen y la difusión del nacionalismo. México: Fondo de Cultura Económica.

Aramburu, M. (2002). Los comercios de inmigrantes extranjeros en Barcelona y la recomposición del “inmigrante” como categoría social. Scripta Nova. Revista Electrónica de Geografía y Ciencias Sociales, VI (108). En http://www.ub.es/geocrit/sn/sn-108.htm. Accedido el 24 de junio de 2009.

Beck, U. (1998). ¿Qué es la globalización? Falacias del globalismo, respuestas a la globalización. Barcelona: Paidós.

Belli, S. y Gil, A. (2009). Llorar en un locutorio: emociones compartidas y consumidas. Comunicación presentada en el IV Congreso de la Cibersociedad. Congreso virtual, del 12 al 29 de noviembre de 2009.

Beltrán, J.; Oso, L. y Ribas, N. (Coords.) (2006). Empresariado Étnico en España. Madrid: Ministerio de Trabajo y Asuntos Sociales y Fundació CIDOB.

Billig, M. (2006) [1995]. Nacionalisme Banal. València: Editorial Afers y Publicacions de la Universitat de València.

Billig, M. (1998). El nacionalismo banal y la reproducción de la identidad nacional. En Revista Mexicana de Sociología, 60(1): 37-57.

Castells, M. (2000). La era de la información. Vol. I. La sociedad red. Madrid: Alianza Editorial.

Castro, A.L. y Zepeda, B.E. (2004). El café Internet: un espacio real para la virtualidad. Jóvenes y ciberespacio en el noroeste de México. Región y Sociedad, septiembre-diciembre, XVI(31): 163-208.

Cavalcanti, L. (2004). La influencia de las nuevas tecnologías en el retorno de los inmigrantes contemporáneos. Scripta Nova. Revista Electrónica de Geografía y Ciencias Sociales, VIII, 170(38). En http://www.ub.es/geocrit/sn/sn-170-38.htm. Accedido el 27 de junio de 2009.

Chao, C.Y.; Wang, P.C.; Lin, Y.M. y Lee, Y.W. (2005). Research on relationship between the behaviour in internet Cafe-Cyberia and social skills for junior high school students of Taiwan. 9th Baltic Region Seminar on Engineering Education. UNESCO International Centre for Engineering Education, Gdynia, Poland.

Chawla, D. y Behl, R. (2006). Perception study of cybercafe users. Global Business Review, 7: 17-41.

De la Haba, J. y Santamaría, E. (2004). De la distancia y la hospitalidad: consideraciones sobre la razón espacial. Athenea Digital, 5: 124-134. En http://antalya.uab.es/athenea/ num5/delahaba.pdf. Accedido el 5 de marzo de 2010.

Delgado, M. (1998). Diversitat i integració. Lògica i dinàmica de les identitats a Catalunya. Barcelona: Empúries. 
Feliu, J. (2004). Les cultures no existeixen. Athenea Digital, 5, 25-34. En http://psicologiasocial.uab.es/athenea/index.php/atheneaDigital/article/view/115/115. Accedido el 25 de setiembre de 2010.

Ferlander, S. y Timms, D. (2006). Bridging the Dual Digital Divide: A Local Net and an ITCafé in Sweden. Information, Communication \& Society, 4(2): 137-159.

Finquelievich, S. y Prince, A. (2007). El (Involuntario) rol social de los cibercafés. Buenos Aires: Dunken.

Garay, A.I.; Martínez, L.M. y Peñaranda, M.C. (2004). Habitando en los locutorios: prácticas cotidianas e inmigración. En C. Serra y J.M. Palaudàrias (Eds.). Actas del IV Congreso sobre Inmigración en España. Girona: Universitat de Girona.'

Gil, A. y Vall-llovera, M. (Eds.) (2006). Jóvenes en Cibercafés. La dimensión física del futuro virtual. Barcelona: Editorial UOC.

Gómez, R.; Hunt, P. y Lamoureux, E. (1999). Telecentros en la mira: ¿Cómo pueden contribuir al desarrollo social? En http://www.bibliotecnic.org/archivos-biblioteca/doc20.pdf. Accedido el 9 de mayo de 2005.

Gürol, M. y Sevindik, T. (2006). Profile of Internet Cafe users in Turkey. Telematics and Informatics, 24: 59-68.

Hong, J. y Huang, L. (2005). A split and swaying approach to building information society: the case of Internet cafes in China. Telematics and Informatics, 22: 377-393.

Íñiguez-Rueda, L.; Martínez, L.; Muñoz-Justicia, J.; Peñaranda-Cólera, M.C. y VitoresGonzález, A. (2012) Telecenters as Association Stations: The Role of Information and Communication Technologies in Migratory Processes. Migraciones Internacionales, Vol. 6 (4): 75-105..

Laegran, A.S. y Stewart, J. (2003). Nerdy, Trendy or Healthy? Configuring the Internet Café. New Media Society, 5: 357-377.

Lee, S. (1999). Private uses in public spaces. A study of an internet café. New media \& Society, vol. 1(3): 331-350.

Liff, S. y Steward, F. (2003). Shaping e-access in the cybercafé: networks, boundaries and heterotopian innovation. New Media Society, 5: 313-334.

Loth, B. (2001). Internet Cafés in Tanzania: a study of the knowledge and skills of endusers. Information Development, 17(4).

Martínez, L.M. y Peñaranda, M.C. (2005). Inmigración y construcción de espacios transnacionales a través del uso de las TIC's. En Psicología Social y problemas sociales. Psicología Política, Cultura, Inmigración y Comunicación Social. En J.M. Sabucedo, J. Romay y A. López-Cortón, Comps. Madrid: Biblioteca Nueva.

Martínez, L.M.; Peñaranda-Cólera, M.C.; Vitores, A. e Íñiguez-Rueda, L. (2011). Los locutorios como espacios de integración: las tecnologías de la información y la comunicación en la construcción de redes e identidades. Psicoperspectivas. Individuo y Sociedad, Vol. 10 (1).

Mauta, T. y Mutula, S.M. (2004). Use of cybercafés: study of Gaborone City, Botswana. 
Program: electronic library and information systems, 38(1): 60-66.

Miller, D. (2004). Etnografia on e off-line: Cibercafés em Trinidad. Horizontes antropológicos, 21: 41-65.

Moya, M. y Álvarez, C. (2007). Mundo Locutorio. Una investigación antropológica en el ciber. En http://weblogs.clarin.com/mundolocutorio/. Accedido el 1 de junio del 2010.

Parella, Sònia (2004). Estrategias étnicas de los comercios étnicos en España. El caso de la ciudad de Barcelona. Actas del IV Congreso sobre la Inmigración en España. Ciudadanía y Participación. Girona, 10-13 de noviembre de 2004.

Peñaranda, M.C. (2005). El locutorio como espacio social transnacional: una mirada psicosocial. Trabajo de investigación no publicado. Departamento de Psicología Social. Universidad Autónoma de Barcelona.

Peñaranda, M.C. (2008). ¿Tecnologías que acercan distancias? Sobre los "claroscuros" del estudio sobre la(s) tecnología(s) en los procesos migratorios transnacionales. En E. Santamaría (Ed.). Retos Epistemológicos de las Migraciones Transnacionales (pp.133164). Barcelona: Anthropos.

Peñaranda, M.C. (2010). "Te escuchas aquí al lado“. Usos de las tecnologías de la información y la comunicación en contextos migratorios transnacionales. Athenea Digital, 19: 239-248.

Peñaranda, M.C.; Vitores, A.; Martínez, L.M.; Muñoz-Justicia, J. e Íñiguez-Rueda, L. (2011). El acceso público a las tecnologías de la información y la comunicación: el lugar de los locutorios en los procesos migratorios. Papeles del CEIC, vol. 2011/1 (70), CEIC (Centro de Estudios sobre la Identidad Colectiva), Universidad del País Vasco. Disponible en http://www.identidadcolectiva.es/pdf/70.pdf. Accedido el 2 de mayo de 2012.

Richer, J. y Doré, J.P. (2004). Téléboutiques. Une forme de sociabilité urbaine en emergence. Flux, núm. 58 : 79-84.

Robertson, R. (1992). Globalization. Social Theory and Global Culture. London: Sage.

Schiavo, T. (2004). Las TIC y sus territorialidades. El caso de los “locutorios” en Quilmes, Metrópolis de Buenos Aires. Comunicación presentada en el Coloquio Internacional “Imaginarios, lugares y metrópolis”, México D.F., 17-19 de octubre de 2004

Scopsi, C. (2002) Commerce ethnique et nouvelles technologies à Barbès : l'émergence d'un nouveau commerce. CRIS/SERIES. Université Paris X-Nanterre. En http://www.jm.upsud.fr/ adis/rubriques/p/jdoctic/scopsi.pdf. Accedido el 3 de abril de 2007.

Scopsi C. (2004). Représentation des TIC et multiterritorialité: Le cas des télé et cyber boutiques de Château-Rouge à Paris. En Technologies de la communication et mondialisation en Afrique. T. Chéneau-Loquay, Dir. Paris: Karthala MSHA. Accedido el 7 de abril de 2005.

Serra, P. (2005). El comercio étnico en el distrito de Ciutat Vella de Barcelona en 2004. Barcelona: Fundación Paulino Torras Domènech, Departamento de Cultura.

Serra, P. (2006). El comercio de los inmigrantes en el centro histórico de Barcelona en 2004. 
Estudios Geográficos, LXVII, 261: 635-672.

Smith, A.D. (1990). Towards a Global Culture? En Global Culture: Nationalism, Globalization and Modernity. M. Featherstone, Ed. Londres: Sage.

Taylor, C. (1992). El multiculturalismo y "la política del reconocimiento". Ciudad de México: FCE.

Taylor, S. y Bogdan, R. (1987) [1984]. Introducción a los métodos cualitativos de investigación. México: Paidós.

Tomlinson, J. (1999). Globalization and Culture. Oxford: Polity Press.

Wakeford, N. (2003). The embedding of local culture in global communication: independent internet cafés in London. New Media Society, 5(3): 379-399. 